\title{
The Recurring Problem of the Third Man
}

\author{
STEVEN D. HALES
}

Brown University

Introduction

The literature on the third man argument is voluminous, and the existence of yet another paper on this topic must be well justified. 1 Pretty much everyone agrees that Plato must have relied on three assumptions in his regress, which have become known as the One Over Many, Self Predication, and Non Identity assumptions. Most writers take Non Identity to be a largely uncontroversial matter of stipulation. The main virtue of this paper is that it argues that Non Identity is consistent with Plato's explicit premises only if Plato accepted a view in the theory of reference which I believe he would have denied. If NI is rejected, the third man argument is not a legitimate regress and Plato's middle period theory of forms has nothing to fear from it. It is also argued that the third man argument is a purely an exercise in ontology, and is not concerned (primarily) with reference or language. The paper is divided into three main sections. The first section is a reading of what happens in the Parmenides before the first third man argument at 132a. The second section presents two interpretations of the third man. The third section is a discussion of reference necessary for evaluation of the two versions of the third man argument.

The Parmenides Before The First Third Man Argument:

I should say that I am assuming that Plato is an extreme realist concerning universals. He considers his forms to be what are now called abstract intensional objects; that is, objects like properties, relations, and numbers. 2 This is not all he believes them to be, of course, but at least part. The translation I have worked from is Cornford's.

1 Some of the especially pertinent literature includes Gregory Vlastos, "The Third Man Argument in the Parmenides", The Philosophical Review, vol.LXIIl, no.3 (1954); Wilfrid Sellars, "Vlastos and the Third Man", Philosophical Review, vol.LXIV, no.3 (1955); P.T. Geach, "The Third Man Again", Philosophical Review, vol.LXV (1956); and Colin Strang, "Plato and the Third Man", reprinted in Plato I: Metaphysics and Epistemology, (ed. G. Vlastos, Notre Dame, 1971).

2 R.E. Allen says that this is the generally accepted view, although he himself disagrees with it. See his "Participation and Predication in Plato's Middle Dialogues", reprinted in Plato I, ibid, p.177. Also compare Vlastos, "Postscript to the Third Man: A Reply to Mr. Geach", Philosophical Review, vol.LXV (1956), p.90, and Lynne Spellman, "Patterns and Copies: The 
126a-127e Introduction

127 -128e Zeno gives a speech and Socrates questions him about it.

128e-130a Socrates: There are the forms likeness and unlikeness, and the many particulars exemplify them. Things can be alike in some ways and unalike in others. Thus the very same object can exemplify both likeness and unlikeness. The same thing is true of unity and plurality. I exemplify unity since I'm just one thing, but insofar as I have parts, I exemplify plurality too. But this doesn't show us anything about the forms themselves. I seriously doubt that the form of unity (e.g.) can exemplify both unity and plurality in the way $I$ can. At any rate, it is a further question. 3 .

130b Parmenides: You're a realist concerning abstract objects, then, Socrates?

Soc: Yes.

Parm: Do you affirm forms for rightness, beauty, and goodness?

Soc: Yes.

130c Parm: What about forms for man, fire, and water?

Soc: I'm unsure about these things.

130d Parm: What about hair, mud, and dirt?

Soc: No, it seems to me that we don't need to posit forms for natural kinds like mud and hair, since the particulars are adequate paradigms in these cases. It is uncontroversial what mud is, while very contentious what

Second Version of the Third Man", Pacific Philosophical Quarterly, 64 (1983), p.174.

3 Plato's actual language concerning this possibility is somewhat stronger. He claims he would be filled with admiration and astonishment if someone could prove that the form of unity exemplifies both unity and plurality. I should also note that I am assuming that the relation of "partaking" or "participation". that the particulars bear to the forms is what is now called "exemplification". Of course, Plato considers relations like "being larger than" and "being equal to" to be forms, and relations aren't exemplified, but are borne. How the middle period Plato can consider relations to be unitary forms is quite a large issue. Geach famously thinks that the form of the equals is a pair of absolutely equal things. In any case, I think it is best to stick with talk of particulars exemplifying forms, rather than muddy up the waters with additional talk of particulars bearing relations (which are forms) to other particulars. I don't see that any philosophical ice would be cut by doing so. 
beauty is. Our belief in forms is only fueled by our need for epistemic paradigms. 4

130e Parm: You'll be more generous in your ontology when you get older and admit forms for natural kinds too.

131a In any case, you agree that there is a relation of exemplification $X$ particulars bear to the form of $X$ ? For example, just things exemplify justice?

Soc: Certainly.

Parm: When a form inheres in a particular (where inherence is the converse of exemplification), is it the whole form which inheres, or a proper part of it? Or shall we analyze exemplification and inherence in some other way?

Soc: No, this way.

Parm: So is it the whole form which inheres or what?

Soc: Why couldn't the whole form inhere in each particular?

131b Parm: One reason is that the whole form would then be in non identical objects at the same time, and one thing can't be in two places at once.

Soc: I think you are wrong here. A form is more like a day, which can be in many places at the same time and is nonetheless just one thing.

Parm: I like that analogy. It is like spreading a sail over a number of people and saying that one sail as a whole was over them all. Is this a fair interpretation?

Soc: Perhaps it is.

131c Parm: Is the sail as a whole over each man, or only a part over one, and a part over another?

Soc: Only a part over each.

Parm: To stick with the analogy, then, only part of a form inheres in each of its instances, contrary to what we had assumed.

Soc: I suppose that's right.

$4 \mathrm{My}$ rendition of this speech is well beyond the actual text. I think it is a better and more consistent response for Socrates than the one he actually makes, which is just to admit confusion over why there are no forms for mud, etc. Compare Euthyphro 7b-7d where Socrates claims that it is relatively easy to, e.g., discover which things are lighter or heavier in weight, but that it is very difficult to discover the nature of right, wrong, and the good. Also see Phaedrus 263a, where Socrates says, roughly, that we all know what iron or silver is, but we dispute about what justice or goodness is. Likewise, cf. Republic VII 523d. 
Parm: Are you prepared to admit that the forms can be divided into parts? Then forms will exemplify both unity and plurality, just as you claimed you do, Socrates. This view yields an answer to your previous question [129c]: the form of unity will exemplify both unity and plurality.

Soc: You're right, it would no longer be just one thing.

Parm: Consider this. A proper part of $X$ is smaller than $X$, by definition. Every large particular is large 131d by possessing a proper part of the form of largeness, by assumption. Thus every large particular is large by possessing something smaller than the form of largeness itself.

Soc: That seems reasonable.

Parm: Take an instance of equality. Since that thing has just a small part of the form of equality, will it be equal to something else?

Soc: No, that's impossible.

Parm: Ok, I have a better example. Consider the form of smallness. The proper parts of smallness will be 131e smaller than smallness itself. Thus the instances of smallness will all have something smaller than smallness itself.

Soc: That's impossible. We seem to have constructed a reductio against the "exemplification as having a proper part of a form" view.

Parm: So if neither the whole form, nor a proper part of it inheres in its instances, how are we to understand inherence and exemplification?

Soc: I am at a loss as to how to analyze these concepts.

Parm: Again, there is another question.

The structure is this. First Socrates questions Zeno about his speech, and makes some claims about the forms. Then Parmenides questions Socrates about what Socrates's position on the forms is. The real discussion starts at 131a. At 131a, Parmenides proposes that to exemplify a form, a thing must possess it in some sort of (still unexplained) physical way. Socrates agrees that this is a good way of viewing exemplification. Two ways of possessing are laid out: possessing the whole form, and possessing a proper part of the form. They decide the former way fails on the basis of Socrates's analogy with the sail. Then from 131d-131e Parmenides gives the following argument against the latter way, and Socrates assents that the argument is sound. 
1) Suppose that small individual objects exemplify the form of smallness by possessing a proper part of the form of smallness. than $X$.

2) For all things $X$ and $Y$, if $Y$ is a proper part of $X$, then $Y$ is smaller

3) Nothing is smaller than the form of smallness.

4) Thus the form of smallness cannot have proper parts. smallness.

5) Thus no individual object can possess a proper part of

6) Thus exemplification is a different relation than the "possessing a proper part of" relation.

Premise (1) is the primary assumption to be falsified by reductio, and (2) follows analytically from the definition of a proper part. Part of the importance of this argument is that in premise (3) we see the first appearance of a Self Predication thesis: the form of smallness is itself small. Moreover, (3) claims that the form of smallness is (necessarily) the smallest thing there is. The conclusions in (4), (5), and (6) follow validly from the premises. (3) is, of course, the questionable premise. How can an abstract object like a form have a physical property like being small? I think that Plato confuses properties (the abstract intension of a predicate) and tropes (a particular exemplification of a property) in his analysis of exemplification, but I will not pursue this hunch here. R.E. Allen has claimed that legitimate universals do not instantiate themselves, and that it is absurd to think otherwise. 5 This is not right. At least some properties do exemplify themselves. For example, being a property is a property, being an abstract object is an abstract object, being eternal is eternal, being capable of being thought of is capable of being thought of, and so on.

There is another argument against the exemplification- ashaving-a-proper-part-of-a-form view, and one catches a whiff of it at 131c. At $129 \mathrm{~d}$, Socrates claims that he exemplifies both unity (since he is one thing) and plurality (since he has many parts). By analogy, if the forms have proper parts, they will likewise exemplify unity and plurality. Around $129 \mathrm{c}$, Socrates claims that he seriously doubts that the form of unity can exemplify both unity and plurality. If it could, then the forms would be no better than the particulars as epistemic paradigms. Both forms and particulars would have various opposing qualities mixed in. Forms are posited as paradigms to start with because they are supposed to be pure and unconfusing. See for example Republic V 479a-480a, and the discussion of equal sticks and stones starting at Phaedo 74b. If Plato were forced to admit that the forms have proper parts, it would be as damaging to his paradigmatic theory of knowledge as the third man argument itself is usually taken to be. More so, perhaps, since the problem is more obvious.

5 Allen, op cit., p.167. 
At Republic V 476a, there is some suggestion that Plato thinks that to exemplify a form is to have some proper part of the form. However, I think that he decisively rejects this view not only in the Parmenides, but also in the Sophist at 245a-b, and in the Phaedo at 78c-e.

1 have argued thus far that the discussion of Parmenides and Socrates up to the first third man argument is a purely ontological one, about how forms relate to their instances. Socrates and Parmenides have concluded that (i) particulars do not physically possess whole forms of which they are instances, (ii) forms do not have proper parts, and (iii) they have not yet correctly analyzed exemplification.

\section{The Third Man Argument}

At the end of 131e, Parmenides seems to set aside analyzing exemplification and move to another point. "Again there is another question...", he says, "how do you feel about this?" Here is Cornford's translation of Parmenides's first speech at 132a.

I imagine your ground for believing in a single form in each case is this. When it seems to you that a number of things are large, there seems, I suppose, to be a certain single character which is the same when you look at them all; hence you think that largeness is a single thing.

This speech is supposed to contain the famous One Over Many Thesis. Socrates nods assent to OM. Parmenides goes on:

But now take largeness itself and the other [large] things which are large. Suppose you look at all these in the same way in your mind's eye, will not yet another unity make its appearance-a largeness by virtue of which they all appear large?

This passage is supposed to contain a Self Predication assumption. Socrates continues to walk down the garden path and agrees to SP. Parmenides moves in for the kill.

If so, a second form of largeness will present itself, over and above largeness itself and the things that share in it, and again, covering all these, yet another, which will make all of them large. So each of your forms will no longer be one, but an indefinite number.

The well known Non Identity thesis, i.e. the claim that the forms spun out by the third man argument are all distinct from each other, is a premise suppressed in Plato's argument and not explicit in the text.

I think there are three ways of viewing the third man argument:

i) as a purely ontological argument.

ii) as a purely linguistic argument.

iii) as a mixed argument about reference. 
If the third man argument is purely ontological, then we might formulate the One Over Many Thesis thus.

$O M_{1}$ : If there are $G$ things, then we can make a set of them, and there is a form which the members of the set all exemplify.

If the third man argument is purely linguistic, then One Over Many is better rendered as

$\mathrm{OM}_{2:}$ If there are subjects in a language $L$, which are subjects of the predicate $F$, then there is the predicate $F$ itself. If the predicate $F$ did not exist in $L$, then it is clear that no subject could be called $F$ in $L$. Thus a necessary condition for $F$ to be predicated of any subject in $L$ is the existence of $F$ in $L$.

If the third man argument is a mixed argument about reference, then One Over Many becomes

$\mathrm{OM}_{3}$ : If one can truly predicate $\mathbf{G}$ of some things (i.e. $\mathbf{G}$ has a non-empty extension) then we can make a set of these things, and there is a form, which is the intension of the predicate $G$.

I think that we can go ahead and rule out the purely linguistic interpretation. Whatever else Plato is up to, he is talking about forms, which are not linguistic entities. He is not giving syntactical rules of grammar, or metalinguistic rules for languages. I list the pure linguistic version for the sake of completeness. Thus the third man argument is either about entia exemplifying forms (ontological version), or it is about predicates in a language mapping into forms (referential version). As a preliminary step to adjudicating between these alternatives, I think we would do well to spell out the third man argument, first in purely ontological language, then in referential language. If one brand of terminology is inadequate for formulating a plausible argument, then that constitutes good grounds for rejecting that terminology. Hopefully it will not be the case that both brands are inadequate.

\section{Ontological Third Man Argument}

OM (1) If there are $G$ things, then we can make a set of them, and there is a form which the members of the set all exemplify.

SP (2) This form itself is $G$.

premise (3) There are large things.

by 1,3 (4) We can make a set of these things and call it s1.

by 1,2 (5) There's a form the members of $s 1$ all exemplify called $f_{1}$.

by $2,5 \quad(6) f_{1}$ is large.

by $4,6 \quad$ (7) $f_{1}$ and the members of $s_{1}$ are large things.

by 1,7 (8) Thus we can make a set of these things and call it s2. 
by 1,7 (9) There's a form, let's call it $f_{2}$, that all the members of $s_{2}$ exemplify.

by $2,9 \quad(10) f_{2}$ is large.

by 9,10 (11) $f_{2}$ and the members of $s 2$ are large things.

(12) Steps 7 and 11 are logically analogous, and

the procession of the regress should be

obvious.

This is the argument as explicitly presented in the Parmenides. I believe that the argument is valid, and clearly so. If you have one form, then you seemingly have an infinite number. As it stands, however, this argument is open to a strong objection, viz., why should we believe that we have an infinite number of different forms? Consider: by premises (3) and (4) we know that there are large things, and that we can make a set of them called $\mathrm{s}_{1}$. By (2) we know the form $\mathrm{f}_{1}$ is also a large thing. Either $\mathrm{s}_{1}$ contains all of the large things, or it only contains some of them. If it contains all the large things, then it contains $f_{1}$. Since $s_{2}$ is defined as the set containing the form $f_{1}$ plus all the members of $s_{1}$, sets $s_{1}$ and $s_{2}$ will have exactly the same members. Both sets will have $f_{1}$ and all the large particulars as their only members. The form that the members of $s_{2}$ all exemplify will be the same form that the members of $s_{1}$ exemplify (since $\left.s_{1}=s_{2}\right)$, viz. $f_{1}$. Sure step (8) of the argument calls the new form $f_{2}$ but we can see that it is the very same form as $f_{1}$. In fact, s1, s2,..., $s_{n}$ turn out to be just be different names for the same set, and $f_{1}, f_{2}, \ldots, f_{n}$ are merely different names for the same form. The third man argument has force only if the forms are diverse. And they are not diverse. Let us call this argument the Collapsing Argument-the iterated forms all collapse to one.

It is in response to this argument that Plato needs the suppressed assumption of Non Identity. Non Identity can be defended on the grounds that $f_{1}$ and $f_{2}$ are different because they have different instances. A necessary condition for two forms to be identical is that they have the same instances at the same time. $f_{1}$ has only the members of $s_{1}$ as instances. (We are tacitly assuming that only individual objects are in s1-but there is nothing logically suspect in this assumption. We can thereby rule $f_{1}$ out of $s_{1}$ by fiat. That is, $s_{1}$ does not contain all of the large things, only the large particulars.) Notably $f_{1}$ does not have itself as an instance. $f_{2}$, on the other hand, has not only the members of $s_{1}$ as instances, but it also has $f_{1}$ as an instance. Hence $f_{1}$ and $f_{2}$ must be different forms. To generalize, $\sim\left(f_{n}=f_{n+k}\right)$ where $n$ and $k$ are non-zero integers. 6 This is the

6 As M. Richard Diaz has pointed out, (in "What is the Third Man Argument?", Southern Joumal of Philosophy, vol.XVI, no.3 (1978), p.157) for the Third Man argument to be successful it must be shown that each new form produced by the regress is non identical to every previous form, not 
hidden premise that Plato needs. But note the commitments of NI. For NI to be consistent with the explicit premise of SP, we cannot interpret SP as claiming that $f_{1}$ exemplifies itself. If $f_{1}$ exemplified itself, then $f_{1}$ would have itself as a instance, and we would lose our grounds for claiming that it isn't identical with $\mathbf{f}_{2}$. And thus we would lose our grounds for accepting NI. What is being claimed by SP, rather, is that $f_{1}$ is large. In other words, a logical wedge must be driven between $f_{1}$ being large and $f_{1}$ exemplifying itself in order for NI to be both true and consistent with SP.

The third man argument is a legitimate regress and generates an infinite number of different forms only if the Collapsing Argument is unsuccessful. The Collapsing Argument is unsuccessful only if NI (i.e. $\sim\left(f_{n}=f_{n+k}\right)$ where $n$ and $k$ are non-zero integers $)$ is true. NI is true-and consistent with SP-only if $f_{1}$ (the form of largeness) can be large and yet not exemplify itself.

So in order to save Plato's third man argument, we must show how it is possible for $f_{1}$ to be large and yet not exemplify itself. Claiming that it is possible seems to buy into a kind of logical muddle that a clear thinker should avoid. There does seem to be one way of defending the idea that $f_{1}$ is large but does not exemplify itself, however. Suppose the predicate 'being large' has more than one intension. Since forms are the intensions of predicates, 'being large' would map to more than one form. There would then be several diverse forms which corresponded to the predicate 'being large'-let's call them $f_{1}, f_{2}$, and $f_{3}$. The form $f_{1}$ could be large (by exemplifying $f_{2}$, say) and yet not exemplify itself. There is no free lunch, though. Taking this route requires buying into certain semantic theories which may be false, distasteful to Plato, or both.

Discussion of these semantic commitments will be more appropriate after I have constructed the third man argument using referential language. Therefore further evaluation of the Ontological Third Man argument and the issues surrounding NI will be temporarily postponed. In the referential version I will proceed on the assumption that the crucial relation in the third man is not that which holds between particulars and forms, but that which holds between predicates and forms. The relation of choice will no longer be ' $x$ exemplifies $y$ ', but ' $y$ is the intension of $x$.

Referential Third Man Argument

OM (1) If one can truly predicate $G$ of some things

just non identical to the last one on the list. It is worth demonstrating that NI as I have formulated it will accomplish this. Pick any form on the regress you like, say, $f_{87} . f_{87}$ will have $f_{86}, f_{85}, f_{84}, f_{83} \ldots, f 1$, plus all the large particulars as instances. No lower number form will have all the same instances as $\mathbf{f 8 7}$, since no form has itself as an instance. Thus each new form is non identical to every previous form. 
(i.e. $\mathrm{G}$ has a non-empty extension) then we can make a set of these things, and there is a form, which is the intension of the predicate $G$.

SP (2) This form itself is $G$.

premise (3) There are large things; i.e. the extension of the predicate 'being large' is non-empty.

by 1,3 (4) We can make a set of the things in the extension of 'being large' and call it 81 .

by 1,2 (5) There's a form called $f_{1}$ which is the intension of the predicate 'being large'.

by 2,5 (6) $f_{1}$ is large.

by 4,6 (7) $f_{1}$ and the members of 81 are large things.

by 1,7 (8) Thus we can make a set of these things, call it

s2.

by 1,7 (9) There's a form called $f_{2}$ which is the intension of the predicate 'being large'.

by 2,9 (10) $f_{2}$ is large.

by 9,10 (11) $f_{2}$ and the members of 82 are large things.

(12) Steps 7 and 11 are logically analogous, and the procession of the regress should be obvious.

This argument is likewise obviously valid. As it stands it is open to the same sort of Collapsing Argument as was leveled against the ontological version. Can the Collapsing Argument be staved off by an appeal to non-identity? Non-identity was defended in the ontological version on the grounds that, given certain assumptions, the iterated forms have different instances. Unfortunately, this line of (ontological) defense is not available here. We need referential grounds to defend non-identity. One way out is to say that 'being large' has diverse meanings; i.e. it has different intensions. Thus $f_{1}$ and $f_{2}$ are different intensions of the same predicate, 'being large'. Note that this is precisely the same stratagem needed to save NI in the ontological version. This I think is a remarkable result. No matter how we interpret the third man argument, the argument is a successful attack on the middle period theory of forms only if Plato subscribes to a certain view in the theory of reference. Plato must hold that it is possible for a predicate to have more than one form as its intension for either version of the third man to work.

Now there are two questions: (1) should Plato have held that a predicate can have more than one form as its intension, and (2) did he hold it? I will examine the first question first. To do so requires a little excursion into the theory of reference.

\section{A Field Trip Into Reference}

At first it seems obvious that one predicate can have different intensions. For example, 'being a ball' can concern either a rubber thing which bounces, or it can connote a formal dance. Predicates like 'being 
large' or 'being flat' may even have an infinite number of intensions. The problem with 'being flat', e.g., is that we preanalytically want to say things like 'west Texas is flat', and 'this desktop is flat'. However, if you look closely enough, everything has bumps. There isn't anything which is absolutely flat. Are our preanalytic claims just false, strictly speaking? And consider this: compared with the desktop, west Texas is not flat. So is the desktop flat and not west Texas, even loosely speaking? In short, we need some account of how to truly apply the predicate 'being flat'. Here is a sketch of some options:

i) Nothing is flat since everything has bumps. The extension of 'being flat' is empty:

ii) Criteria for inclusion into the extension of 'being flat' are context relative. When we're talking about land, west Texas is in the extension of 'being flat'. When we're talking about desks, west Texas is not in the extension of 'being flat', since compared to a desktop, west Texas is not flat. There is just one predicate 'being flat', and its extension varies across contexts.

iii) 'Being flat' becomes a different (albeit homonymic) predicate when we switch to another context. For every context $n$, 'being flat $n^{\prime}$ ' has fixed, definite criteria for forming its extension. When we are talking about desktops being flat, we are really using the predicate 'being flatdesktop'. When we are talking about west Texas being flat, we are really using the predicate 'being flatland'. There is a different homonymic predicate 'being flat $n$ ' for every context $n$.

Option (i) is endorsed by Peter Unger (at least at one time). ${ }^{7}$ The advantage of (i) is that the criteria for getting into the extension are fixed and specifiable, and thus 'being flat' has only one intension. A disadvantage of (i) is that nothing in this world is flat, and so 'being flat' becomes a pretty useless predicate (at least outside of fiction). We will need some other less strict predicate to describe our desktops and land. I wonder what kind of possible world actually contains flat things. A world with only monads as individual objects? $A$ world with only two spatial dimensions? I don't know.

Option (ii) is taken by David Lewis. 8 The advantage of (ii) is that it allows us to talk about flat things in this world, and make sense of the fact that west Texas and the desktop are both flat, but not "in the same breath". The disadvantage of (ii) is that the criteria for the extension of 'being flat' change from context to context. A necessary condition for intension

7 Unger, Peter, Ignorance, (Oxford: Oxford University Press, 1975), pp.6568. In a more recent work, Unger seems to be taking a mellower position. See his Philosophical Relativity (Minneapolis: University of Minnesota Press, 1984), pp.22-23.

8 Lewis, David, "Scorekeeping in a Language Game", Journal of Philosophical Logic, 8 (1979), esp. p.353. 
identity is necessary coextensiveness. When we use 'being flat' in the context of land we get one extension, and when we use it in the context of desktops we get another. Thus 'being flat' has different extensions, and hence it has different intensions. If for every distinct context we get a correspondingly distinct extension for 'being flat', and there are an infinite number of different contexts, then there are an infinite number of different intensions for 'being flat'.

I do not know of anyone who explicitly embraces option (iii), but I wish to submit it as a live possibility. 9 The advantage of (iii) is that for any 'being flat $n$ ', there are fixed and definite criteria for membership in its extension. Thus any 'being flatn' has only one intension. We might call (iii) a hatstand model-one predicate, one intension. As a bonus, (iii) comes with all the advantages of (ii), namely, that we can make sense of the fact that the desktop is flat and west Texas is flat, but not by comparison with the desktop. West Texas is not flatdesktop, but it is flatland. There is no 'being flat simpliciter' according to which the desktop is flat and west Texas is not. If there is a 'being flat simpliciter', then it is the very strict sense of flatness which none of the bumpy material objects in this world can satisfy. The disadvantage of (iii) is that we are committed to admitting that there are many more predicates in our language than we had suspected, and that many of them are (to our confusion) homonymic. Further defense of (iii) is beyond the limits of this paper.

If (iii) is a plausible option, then we need not say that Plato should have held that a predicate can have more than one form as its intension. But was (iii) available to Plato? Would he have been amenable to it? I submit that the answer is yes. Colin Strang thinks the third man argument is a reductio against a uniqueness thesis: there is one and only one form of (e.g.) largeness.10 Why is Plato committed to the uniqueness thesis? A large part of Plato's motivation to believe in the theory of forms is that forms are supposed to be epistemic paradigms. We learn how to apply predicates via our recollection of the forms they express. An epistemic paradigm is supposed to be an explanatory telos; a final, self subsuming answer to the question "what is it?".11 If there is a red tag sale on formsbuy one, get some more for free-then it is hard to see how the "what is it" question will get a satisfactorily final, self subsuming answer. If there is more than one form for 'being large', Plato's paradigmatic theory of learning faces grave difficulties. Anders Wedberg has claimed that in Republic X Plato explicitly rejects the idea that a predicate can have more than one intension. Wedberg writes, "corresponding to each plurality of

9Felicia (formerly Diana) Ackerman may advocate this view. 10 Strang, op cit.

11 By comparison, Sarah Waterlow calls forms "fixed stopping- points of being" in "The Third Man's Contribution to Plato's Paradigmatism", Mind, vol.XCI (1982), p.347. 
objects to which we apply a common name, there is a unique ldea which justifies the application of that name...for each name " $Y$ ", there is a unique Idea such that being $Y$ and participating in that Idea are necessarily the same".12

If Wedberg is right about this, and Plato antecedently denies that it is possible for a predicate to express more than one form, Plato cannot consistently embrace either version of the third man argument. The referential version of the third man is an obvious non starter, since NI is defenseless. If 'being large' can express only one form, then there is no way that the argument can generate an infinite number of diverse forms of largeness. The "forms" ostensibly iterated by the referential version are clearly just different names for the same form. Matters are a little more complicated in the ontological version. NI is, of course, required for the ontological version to be successful. Recall that it was argued that NI is both defensible and consistent with SP only if a logical wedge can be driven between $f_{1}$ (the first form expressed by 'being large' generated by the third man argument) being large and $f_{1}$ exemplifying itself. It was then argued that this wedge can be driven only if 'being large' can have more than one form as its intension. Since Plato rejects this last condition, the ontological version of the third man argument also fails to produce an infinite number of different forms.

If both versions of the third man argument fall to the Collapsing Argument, what grounds do we have for thinking that Plato intended one version rather than the other? I think there are two reasons for holding that Plato intended the ontological version. The first is that, as I have argued, given Plato's antecedent rejection of predicates mapping to more than one form, the referential version of the third man argument cannot get off the ground. I hesitate to saddle Plato with an argument clearly premised on something he rejects. The ontological version fails for basically the same reasons, but how this is so is considerably more opaque. It is reasonable to think that Plato did not see the final commitments required to make the ontological third man work. The second reason I find the ontological version to be the likelier candidate is that accepting the ontological third man as the argument intended by Plato squares well with Socrates's and Parmenides's discussion just prior to 132a. I have previously argued that their discussion was purely ontological, about how particulars relate to forms. While Parmenides at 132a turns away from analyzing exemplification, exemplification is still on his mind when he presents the third man argument. When Parmenides says "again, there is another question" at 131e, he means "again, there is another question about exemplification".

12 Wedberg, Anders, Plato's Philosophy of Mathematics (Stockholm: Almqvist and Wiksell, 1955), chapter 3. 
Conclusion

Plato may honestly have thought that the third man argument was a troubling and powerful objection to his uniqueness thesis and hence the middle period theory of forms. It is no slight on Plato to say that he did believe the third man to be sound. It is far from obvious why the regress fails; a good deal of analysis has been required to show its failings. Regardless of what Plato might have believed about the argument, I am pleased to tell him that he is free to keep forms as universals (the One Over Many Thesis), free to keep forms as paradigmatic instances (the Self Predication Thesis), and free to keep these paradigms as unique ends of explanation (the Uniqueness Thesis). I conclude that insofar as the first third man argument in the Parmenides is concerned, Plato can keep the middle period theory of forms intact. 13

13 I am indebted to Martha Nussbaum for criticisms of an earlier version of this paper, and to James Dreier, John Greco, and Jaegwon Kim for helpful discussions. 